\title{
Necesidades académicas, personales, sociales y vocacionales de estudiantes internacionales e intercambio en la Universidad de Puerto Rico, Recinto de Río Piedras
}

\section{Academic, Personal, Social and Vocational Needs of International and Exchange Students at the University of Puerto Rico, Río Piedras Campus}

\author{
Ángel A. Villafañe Santiago ${ }^{1}$ \\ Recinto de Río Piedras, Universidad de Puerto Rico \\ Puerto Rico \\ angelpr39@hotmail.com \\ Carol Y. Irizarry Robles ${ }^{2}$ \\ Recinto de Río Piedras, Universidad de Puerto Rico \\ Puerto Rico \\ carol_irizarry@live.com \\ Robinson A. Vázquez Ramos ${ }^{3}$ \\ Recinto de Río Piedras, Universidad de Puerto Rico \\ Puerto Rico \\ ravazquezramos@gmail.com
}

Recibido 20 de setiembre de 2011 • Aceptado 24 de octubre de 2011

Resumen. El propósito de este estudio fue identificar las necesidades de una muestra de estudiantes internacionales y de intercambio que cursan estudios en la Universidad de Puerto Rico, Recinto de Río Piedras. Se exploraron datos socio-demográficos así como las necesidades académicas, vocacionales, y personales/sociales, con los fines de recopilar información de relevancia para universidades que sirven esta población y someter recomendaciones que atiendan las necesidades. Los hallazgos revelaron necesidades mayormente en las áreas académicas y vocacionales. Entre estas, se destacan la necesidad de aprender inglés o español, y el conocer sobre internados y ofrecimientos académicos en Puerto Rico y fuera del país, sueldo y oportunidades de empleo, ayudas económicas y servicios estudiantiles en la Universidad de Puerto Rico. Se proveen recomendaciones para trabajar con las necesidades identificadas ya que, según establece la bibliografía, las dificultades que confrontan estos/as estudiantes pueden afectar su desempeño académico e incluso su salud emocional.

Palabras claves. Necesidades, estudiantes internacionales, estudiantes de intercambio, ajuste.

Doctor en Educación con concentración en consejería, de la Universidad Interamericana de Puerto Rico, Maestría en Consejería en Rehabilitación de la Universidad de Puerto Rico y bachillerato en Trabajo Social de la Universidad Central de Bayamón, Puerto Rico. Actualmente se desempeña como Catedrático Auxiliar de consejería en el Departamento de Consejería para el Desarrollo Estudiantil (DCODE) de la Universidad de Puerto Rico, donde se realizan procesos de terapia individual, grupal, consejería vocacional e investigaciones.

Correspondencia: PO Box 23137 San Juan, Puerto Rico 00931-3137. angelpr39@hotmail.com

2 Estudiante doctoral del Programa Graduado de Psicología de la Universidad de Puerto Rico, Recinto de Río Piedras. Miembro de la Asociación de Psicología de Puerto Rico. Ha colaborado en varias investigaciones y su trabajo ha sido presentado a nivel internacional. Actualmente se desempeña como asistente de investigación en el Departamento de Consejería para el Desarrollo Estudiantil de la Universidad de Puerto Rico, Recinto de Río Piedras.

3 Doctor en Filosofía en Educación de Consejería en Rehabilitación de la Universidad de Iowa en Estados Unidos. Maestría en Educación con concentración en Evaluación e Investigación. Actualmente se desempeña como Catedrático Auxiliar en la Escuela Graduada de Consejería en Rehabilitación de la Universidad de Puerto Rico, Recinto de Río Piedras. 


\begin{abstract}
The purpose of this study was to identify the needs of a sample of the international and exchange students staying at the North Tower Residence of the University of Puerto Rico, Río Piedras Campus. Social and demographic data, as well as the academic, vocational, and social/personal needs were explored with the purpose of gathering relevant information and submitting recommendations to universities serving this student population. Findings revealed particular needs in the academic, vocational areas, including English or Spanish learning, information on internships and study programs in Puerto Rico or abroad, salary and employment opportunities, financial aid and services available to the students of this University. Recommendations are provided to work with the identified needs. According to the literature, difficulties faced by these students may affect their academic performance and emotional health.
\end{abstract}

Keywords. Needs, international students, exchange students, adjustment.

\title{
Introducción
}

La cifra de estudiantes extranjeros y extranjeras ha ido en aumento en el recinto riopedrense de la Universidad de Puerto Rico, hecho que coincide con la tendencia de las universidades de otros países (Cheng y Fox, 2008; Gu, Schweisfurth y Day, 2010; Hwang, Wang y Sodanine, 2011; Institute of International Education, 2010; Russell, Rosenthal y Thomson, 2010; Suanet y Van de Vijver, 2009). Para el año académico 1992-1993 se matricularon 68 estudiantes extranjeros y extranjeras, mientras que para el segundo semestre del año académico 2008-2009 esta cifra aumentó a 184 (Universidad de Puerto Rico, Oficina de Planificación Académica, 2009). Esto puede estar vinculado a las políticas de internacionalización promovidas en la Universidad de Puerto Rico en los últimos años, las cuales han propiciado el que estudiantes de otros países cursen estudios en el recinto. Esto provee al estudiantado la oportunidad de ampliar sus experiencias y conocimientos no solo académicos sino también culturales. No obstante, el migrar de un país a otro constituye un cambio radical que puede afectar a la persona en diferentes ámbitos.

Cuando una persona de una cultura se traslada a otra que es diferente, experimenta un choque cultural y puede confrontar dificultades para satisfacer las necesidades básicas, lo cual puede incluso tener un impacto en su bienestar psicológico (Cemalcilar y Falbo, 2008). Las necesidades usuales que tiene se exacerban al confrontar un entorno físico diferente que obliga a realizar cambios en el estilo de vida y a manejar un nuevo sistema de valores, creencias, costumbres y normas sociales (Afflick, 2009). Los estudiantes y las estudiantes internacionales y de intercambio no están exentos de este fenómeno. Al estudiar en otro país, pasan por un periodo de adaptación que varía de acuerdo con las diferentes culturas (Tomich, McWhirter y Darcy, 2003), lo que provoca unas necesidades particulares que deben ser atendidas. Estas pueden variar desde aspectos elementales, tales como el conocer el entorno de la residencia donde se hospedarán y los lugares para obtener artículos de primera necesidad, hasta aspectos más complejos, como el aprender otro idioma y saber cómo interactuar en la universidad y en la comunidad externa.

Siguiendo la teoría de Maslow (1967), las personas nacen con unas necesidades fisiológicas que son innatas o hereditarias. Al principio, su comportamiento gira en torno de la satisfacción cíclica de ellas (hambre, sed, sueño, etc.). Sin embargo, a partir de cierta edad, se comienza un proceso de aprendizaje de nuevos patrones. Surgen aquellas relacionadas con la seguridad enfocada hacia la protección contra el peligro, las amenazas y las privaciones. Las fisiológicas y las de seguridad son consideradas primarias, 
y tienen que ver con su conservación personal. En la medida que se logra controlarlas, aparecen lenta y gradualmente otras más elevadas: sociales de estima, pertenencia y de autorrealización. Por ello, no se puede perder de perspectiva la satisfacción de estas en el contexto académico, de manera que se cuente con los recursos para asumir los retos académicos y alcanzar las metas propuestas.

Por otro lado, es necesario considerar que los estudiantes y las estudiantes internacionales y de intercambio pueden ser recipientes de prejuicios, percibir que se les discrimina (Charles-Tossaint y Crowson, 2010; Nilsson, Butler, Shouse y Joshi, 2008; Poyrazli y Grahame, 2007; Wadsworth, Hecht y Jung, 2008), sentir falta de apoyo social (Sümer, Poyrazli y Grahame, 2008), altos niveles de aislamiento (Erichsen y Bollinger, 2011) y estrés por estar lejos de su familia (Väfors, Chin y DeMarinis, 2008), y por el proceso de aculturación (Nilsson et al., 2008; Ying y Han, 2006). La bibliografía ha señalado que los estresores económicos, sociales y académicos que experimentan pueden, en algunos casos, generar o exacerbar trastornos mentales (Bradley, 2000) y generar depresión y ansiedad (Russell et al., 2010; Ying y Han, 2006), lo cual puede manifestarse incluso en síntomas somáticos (Mori, 2000); esto podría implicar ausencias al salón de clase e ingesta de medicamentos que tienen efectos secundarios. Además, generalmente llegan a través de visados especiales para prepararse académicamente, que demandan el completar los estudios dentro de un tiempo delimitado, lo cual puede ser un estresor adicional.

Muchos y muchas estudiantes internacionales y de intercambio confrontan dificultades que requerirían la intervención de un profesional de ayuda. Sin embargo, la mayoría no la solicita (Mori, 2000). En ocasiones esto sucede debido a que no conocen sobre los servicios disponibles (Hyun, Quinn, Madon y Lustig, 2007); no obstante, estudios han reportado mucha resistencia para hablar sobre sus problemas y recibir servicios de consejería (Bradley, 2000), en ocasiones por el estigma que algunas culturas adjudican a la necesidad de ayuda terapéutica (Masuda et al., 2009; Zhang y Dixon, 2003). Este panorama es preocupante toda vez que al afectarse su bienestar, también se impacta su desempeño académico (Hyun et al., 2007), por tanto, resulta fundamental que las universidades se les acerquen y atiendan sus necesidades para asistir su proceso de ajuste, disminuir el impacto en su salud y su desempeño académico, y facilitar que puedan aspirar a la autorrealización al alcanzar sus metas académicas.

Reconociendo la importancia de esto, el Departamento de Consejería para el Desarrollo Estudiantil (DCODE) de la Universidad de Puerto Rico, Recinto de Río Piedras, realizó un estudio para identificar las necesidades de una muestra de estudiantes internacionales y de intercambio de este recinto, con los fines de recopilar información de relevancia para las universidades que sirven a esta población, así como para el estudiantado al que le interesa prepararse para participar en programas internacionales o de intercambio, y someter recomendaciones que atiendan sus necesidades.

\section{Método}

\section{Diseño}

El diseño que se utilizó para esta investigación fue uno exploratorio-descriptivo. El elemento exploratorio busca conocer sobre un fenómeno en particular del que se tiene poco o ningún conocimiento (Hernández, Fernández y Baptista, 2003). Al respecto, este estudio constituye uno de los pocos que han explorado el tema en Puerto Rico. 
De forma similar, el elemento descriptivo define la existencia, registra las características e identifica las dimensiones de un grupo de variables asociadas a un fenómeno en particular que ha sido estudiado en un tiempo determinado. A tales efectos, el diseño exploratorio-descriptivo resulta útil y apropiado para este estudio ya que permite obtener un perfil o delinear las necesidades académicas, personales, sociales y vocacionales de un grupo de estudiantes internacionales e intercambio en la Universidad de Puerto Rico, Recinto de Río Piedras.

\section{Muestra}

La muestra de este estudio fue seleccionada por disponibilidad y estuvo constituida por 53 estudiantes (52\%) de un universo de 101 estudiantes del Programa de Estudiantes Internacionales y del Programa de Estudiantes de Intercambio que se hospedaban en la Residencia Torre Norte de la Universidad de Puerto Rico, Recinto de Río Piedras. Se escoge este lugar para la selección de la muestra porque es donde más estudiantes internacionales y de intercambio se hospedan al matricularse en la Universidad de Puerto Rico.

\section{Instrumento}

El instrumento que se utilizó consistió de dos partes. La primera fue una planilla de datos demográficos relacionados con: edad, género, estado civil, país de origen, país de ciudadanía, ayuda financiera, tipo de beca y programa de admisión (internacional o de intercambio). También incluyó la facultad o programa graduado, grado que obtendría, tiempo en Puerto Rico, e interés de participar en una organización estudiantil que trabaje por el bienestar de los estudiantes internacionales y de intercambio.

La segunda parte consistió de un instrumento que fue creado tomando de referencia la bibliografía relacionada con las necesidades de estudiantes internacionales entre los años 2000 al 2007 y algunos reactivos de los instrumentos de las siguientes investigaciones: Necesidades $y$ problemas de los estudiantes internacionales matriculados en el Recinto de Río Piedras de la Universidad de Puerto Rico, realizada por Nazario (1992); el Cuestionario de necesidades de orientación de los estudiantes de primer año, del Departamento de Consejería para el Desarrollo Estudiantil de Vélez (2006); el cuestionario de preguntas estructuradas de la investigación Evaluation of Academic and Social Needs of Foreign-Born Students at a University and School of Nursing realizada por Dorough (2006) y presentada como su disertación en Nova Southeastern University; y algunos reactivos de la investigación titulada A Comparative Analysis of Foreign Student Needs in Taiwan de Lee (2000).

Luego del análisis de estos instrumentos, decidimos definir necesidad como aquellas dificultades que fueron identificadas por la población de estudiantes internacionales y de intercambio en el proceso de ajuste al nuevo ambiente universitario; importancia fue definida como el grado de interés que otorgaron para que se trabajaran las mismas, medido por niveles de intensidad. Los reactivos se organizaron en tres áreas de necesidad. Estas fueron: académica/educativa con veintidós (22) premisas, la personal/social con veinticuatro (24) premisas y la vocacional con once 
(11) premisas. Debían indicar si tenían o no esa necesidad marcando "sí" o "no". Además, se utilizó una escala para que indicaran si era muy importante, importante o no importante que se trabajara con ella. Este instrumento pasó por un análisis de juicio de expertos para determinar la evidencia asociada a la validez de contenido y la validez aparente. Una vez finalizado, se reestructuraron las premisas según las recomendaciones y se ubicaron en las distintas categorías. Luego, se tradujo al idioma inglés, con el propósito de que aquellos y aquellas estudiantes que no dominaran el idioma español pudieran participar del estudio. El instrumento tuvo un alpha de Cronbach de .92.

\section{Procedimiento}

Se solicitó autorización del Comité Institucional para la Protección de los Seres Humanos en la Investigación de la Universidad de Puerto Rico. Una vez se obtuvieron las autorizaciones pertinentes, se coordinó con los directores auxiliares de la Residencia Torre Norte para ubicar la hoja informativa y el instrumento en inglés y en español en los buzones estudiantiles para que seleccionaran en cuál de los dos idiomas deseaban contestarlo y se les dio una semana para ello. Luego, lo depositaron en un buzón que fue ubicado al lado del sistema de correo donde reciben la correspondencia. De esta manera, se pretendió evitar cualquier tipo de coerción tanto de la institución como del equipo de investigación.

\section{Resultados}

Buscando conocer las necesidades particulares de los estudiantes y las estudiantes internacionales y de intercambio, exploramos sus necesidades académicas, vocacionales, $\mathrm{y}$ personales/sociales así como sus datos socio-demográficos.

\section{Datos sociodemográficos}

De los datos socio-demográficos se desprende que un $64.2 \%$ de estudiantes ingresaron al Programa Internacional y un $35.8 \%$ al Programa de Intercambio. Un 58.5\% son graduados que aspiran a maestría o doctorado, de los cuales la mayoría son internacionales; y un $41.5 \%$ son subgraduados que aspiran a un bachillerato, de los cuales la mayoría corresponde a intercambio. Un $37.7 \%$ fue admitido a la Facultad de Humanidades, $22.6 \%$ a Ciencias Naturales, 35.9\% a otras facultades y $3.8 \%$ no contestó.

El género femenino predominó con un $77.4 \%$ y el $94.3 \%$ indicó ser soltera o soltero. La mayoría se ubicó entre las edades de 20 a 25 años con un 49.1\%, mientras que un 39.6\% tenía entre 26 a 32 años, 5.7\% entre 33 a 39 años, 3.8\% tenía 17 años o menos, y $1.9 \%$ contaba con 40 años o más. Un 30.2\% proviene de Estados Unidos, 24.5\% de Colombia, 7.5\% de Francia, 5.7\% de España, Italia, Perú y República Dominicana cada uno, y el restante 15\% de otros países. De estos grupos, un $32.1 \%$ tenía ciudadanía estadounidense, $22.6 \%$ colombiana y el $54.3 \%$ ciudadanía de su país de procedencia. La ciudadanía correspondió al lugar de procedencia de cada estudiante, con 


\section{URL: http://www.una.ac.cr/educare}

excepción de una estudiante de Colombia que indicó tener ciudadanía estadounidense. Un 47.3\% de estudiantes del Programa Internacional que participaron del estudio provienen de países hispanos.

El estudió arrojó que el 20.8\% no recibe ayuda económica, mientras que el 79.2\% sí la recibe. Un $37.7 \%$ tienen ayuda económica a través de ayudantías de cátedra en la Universidad de Puerto Rico, $18.9 \%$ recibe ayudas financieras del gobierno de los Estados Unidos, un $18.8 \%$ recibe alguna ayuda -ya sea pública o privada- procedente de su país de origen, y un $9.4 \%$ indicó otro tipo de ayuda, incluyendo estudiantes que originalmente habían marcado que no recibían ayuda económica. En cuanto al tiempo de estadía en Puerto Rico, un 35.8\% tenía más de 10 meses en Puerto Rico, 34\% menos de 3 meses, 20.8\% entre 4 a 6 meses, y 9.4\% entre 7 a 9 meses. El estudio exploró el interés de participar en una organización estudiantil que trabaje por su bienestar, encontrándose que el 71.7\% mostró interés en la misma.

A continuación, presentamos aquellas necesidades que un $50 \%$ o más de estudiantes identificaron tener y que consideraron muy importante el que se trabaje con estas. Se debe observar que en las necesidades personales/sociales, un 50\% o más indicaron que era muy importante trabajar esa necesidad, aunque menos del $50 \%$ identificaron tenerla.

Tabla 1

Necesidades y la importancia de trabajarlas

\begin{tabular}{|c|c|c|c|c|c|c|c|c|c|c|}
\hline & \multicolumn{2}{|c|}{ Sí } & \multicolumn{2}{|c|}{ No } & \multicolumn{2}{|c|}{ Muy importante } & \multicolumn{2}{|c|}{ Importante } & \multicolumn{2}{|c|}{ No importante } \\
\hline & $\mathrm{F}$ & $\%$ & $\mathrm{~F}$ & $\%$ & $\mathrm{~F}$ & $\%$ & $\mathrm{~F}$ & $\%$ & $\mathrm{~F}$ & $\%$ \\
\hline \multicolumn{11}{|c|}{ Necesidades académicas } \\
\hline $\begin{array}{l}\text { Desarrollar destrezas en el idioma } \\
\text { inglés. }\end{array}$ & 32 & 64.0 & 18 & 36.0 & 31 & 68.9 & 4 & 8.9 & 10 & 22.2 \\
\hline Conocer los servicios estudiantiles. & 43 & 86.0 & 7 & 14.0 & 31 & 64.6 & 14 & 29.2 & 3 & 6.3 \\
\hline $\begin{array}{l}\text { Entender los libros de texto en } \\
\text { idiomas diferentes al mío. }\end{array}$ & 36 & 69.2 & 16 & 30.8 & 29 & 60.4 & 12 & 25.0 & 7 & 14.6 \\
\hline $\begin{array}{l}\text { Desarrollar destrezas en el idioma } \\
\text { español. }\end{array}$ & 26 & 50.0 & 26 & 50.0 & 24 & 60.0 & 6 & 15.0 & 10 & 25.0 \\
\hline Obtener orientación académica. & 42 & 85.7 & 7 & 14.3 & 28 & 58.3 & 18 & 37.5 & 2 & 4.2 \\
\hline $\begin{array}{l}\text { Aprender a preparar informes } \\
\text { escritos. }\end{array}$ & 27 & 51.9 & 25 & 48.1 & 25 & 56.8 & 11 & 43.2 & 8 & 18.2 \\
\hline $\begin{array}{l}\text { Obtener información sobre los } \\
\text { eventos académicos, servicios y } \\
\text { facilidades universitarias. }\end{array}$ & 36 & 70.6 & 15 & 29.4 & 24 & 55.8 & 18 & 41.9 & 1 & 2.3 \\
\hline $\begin{array}{l}\text { Aprender a preparar un } \\
\text { informe oral. }\end{array}$ & 27 & 51.9 & 25 & 48.1 & 24 & 55.8 & 14 & 32.6 & 5 & 11.6 \\
\hline $\begin{array}{l}\text { Aprender a usar las bibliotecas } \\
\text { (Internet, correo electrónico y } \\
\text { redes de comunicación). }\end{array}$ & 27 & 52.9 & 24 & 47.1 & 22 & 52.4 & 13 & 31.0 & 7 & 16.7 \\
\hline
\end{tabular}

Continúa... 
URL: http://www.una.ac.cr/educare

\begin{tabular}{|c|c|c|c|c|c|c|c|c|c|c|}
\hline & \multicolumn{2}{|c|}{ Sí } & \multicolumn{2}{|c|}{ No } & \multicolumn{2}{|c|}{ Muy importante } & \multicolumn{2}{|c|}{ Importante } & \multicolumn{2}{|c|}{ No importante } \\
\hline & $\mathrm{F}$ & $\%$ & $\mathrm{~F}$ & $\%$ & $\mathrm{~F}$ & $\%$ & $\mathrm{~F}$ & $\%$ & $\mathrm{~F}$ & $\%$ \\
\hline \multicolumn{11}{|c|}{ Necesidades personales/sociales } \\
\hline Manejar el tiempo adecuadamente. & 24 & 47.1 & 27 & 52.9 & 20 & 51.3 & 14 & 35.9 & 5 & 12.8 \\
\hline $\begin{array}{l}\text { Aprender acerca de los ajustes } \\
\text { sociales en la UPR }\end{array}$ & 22 & 44.0 & 28 & 56.0 & 20 & 50.0 & 12 & 30.0 & 8 & 20.0 \\
\hline \multicolumn{11}{|c|}{ Necesidades vocacionales } \\
\hline $\begin{array}{l}\text { Explorar internados local e } \\
\text { internacionalmente relacionados a } \\
\text { mi preparación académica. }\end{array}$ & 38 & 82.6 & 8 & 17.4 & 35 & 77.8 & 8 & 17.8 & 2 & 4.4 \\
\hline $\begin{array}{l}\text { Conocer sueldo y oportunidades } \\
\text { de empleo de mi preparación } \\
\text { académica. }\end{array}$ & 40 & 80.0 & 10 & 20.0 & 33 & 76.7 & 8 & 18.6 & 2 & 4.7 \\
\hline $\begin{array}{l}\text { Conocer en cuáles escenarios } \\
\text { podría desempeñarme con mi } \\
\text { preparación académica. }\end{array}$ & 32 & 65.3 & 17 & 34.7 & 34 & 75.6 & 7 & 15.6 & 4 & 8.9 \\
\hline $\begin{array}{l}\text { Explorar otras universidades en } \\
\text { EU que tengan ofrecimientos } \\
\text { académicos de interés para mi } \\
\text { persona }\end{array}$ & 30 & 66.7 & 15 & 33.3 & 27 & 71.1 & 7 & 18.4 & 4 & 10.5 \\
\hline $\begin{array}{l}\text { Adquirir conocimientos sobre } \\
\text { ayudas económicas disponibles en } \\
\text { la universidad }\end{array}$ & 37 & 75.5 & 12 & 24.5 & 30 & 68.2 & 10 & 22.7 & 4 & 9.0 \\
\hline $\begin{array}{l}\text { Aprender más sobre los } \\
\text { ofrecimientos académicos en el } \\
\text { Sistema de la UPR }\end{array}$ & 31 & 67.4 & 15 & 32.6 & 26 & 65.0 & 11 & 27.5 & 3 & 7.5 \\
\hline $\begin{array}{l}\text { Conseguir un empleo a tiempo } \\
\text { parcial dentro de la universidad }\end{array}$ & 35 & 68.6 & 16 & 31.4 & 29 & 64.4 & 11 & 24.4 & 5 & 11.1 \\
\hline $\begin{array}{l}\text { Conocer las tareas de la ocupación } \\
\text { que me interesa o estoy estudiando }\end{array}$ & 27 & 57.4 & 20 & 42.6 & 26 & 61.9 & 11 & 26.2 & 5 & 11.9 \\
\hline $\begin{array}{l}\text { Explorar mis intereses } \\
\text { ocupacionales }\end{array}$ & 26 & 52.0 & 24 & 48.0 & 23 & 57.5 & 11 & 27.5 & 6 & 15.0 \\
\hline
\end{tabular}

\section{Necesidades académicas}

Respecto a estas, el 68.9\% indicó que es muy importante desarrollar destrezas en el idioma inglés, el $60 \%$ desarrollar destrezas en español y el $60.4 \%$ entender libros de texto en idiomas diferentes al suyo. Un $64.6 \%$ consideró muy importante conocer los servicios estudiantiles, el 58.3\% obtener orientación académica, y el 55.8\% obtener información sobre los eventos académicos, servicios y facilidades universitarias. El 56.8\% indicó además que necesitaban aprender a preparar informes escritos, el 55.8\% que necesitaban aprender a preparar informes orales, y el 52.4\% señaló como muy importante el aprender a usar las bibliotecas, incluyendo el Internet, el correo electrónico y las redes de comunicación. 


\section{Necesidades vocacionales}

En esta área, el $77.8 \%$ señaló que es muy importante explorar internados en Puerto Rico e internacionalmente relacionados con su preparación académica. A esto se añade que el 76.7\% indicó como necesidades muy importantes el conocer el sueldo y las oportunidades de empleo de acuerdo con su preparación académica; el $75.6 \%$ conocer en cuáles escenarios podría desempeñarse con su preparación, el $61.9 \%$ conocer las tareas de la ocupación que está estudiando o le interesa, y el $57.5 \%$ explorar sus intereses ocupacionales. Además, un $68.2 \%$ señaló como muy importante el adquirir conocimientos sobre ayudas económicas disponibles en la universidad y el $64.4 \%$ conseguir un empleo a tiempo parcial dentro de la universidad. Otro dato relevante es que el $71.1 \%$ señaló como muy importante el explorar otras universidades en Estados Unidos que tengan ofrecimientos académicos de su interés, mientras que el $65 \%$ consideró muy importante el que se trabaje con la necesidad de aprender más sobre los ofrecimientos académicos en el Sistema de la Universidad de Puerto Rico.

\section{Necesidades personales/sociales}

La mayoría no indicó tener necesidades en esta área. No obstante, el 51.3\% consideró que es muy importante que se trabaje con la necesidad de manejar el tiempo adecuadamente y un $50 \%$ con la necesidad de aprender acerca de los ajustes sociales en la Universidad de Puerto Rico.

\section{Discusión}

El propósito de este estudio fue identificar las necesidades de una muestra de estudiantes internacionales y de intercambio que cursan estudios en la Universidad de Puerto Rico, Recinto de Río Piedras. Para ello, se exploraron datos socio-demográficos así como las necesidades académicas, vocacionales, y personales/sociales, con los fines de recopilar información de relevancia para universidades que sirven esta población y someter recomendaciones que atiendan las mismas.

\section{Datos socio-demográficos}

Se ha observado que, por lo general, los estudiantes y las estudiantes del Programa Internacional emigran para buscar mejores oportunidades de estudio y obtener un grado universitario en un lugar de prestigio (Hwang et al., 2011; Szelényi, 2006; Wadsworth et al., 2008), por lo que su estadía tiende a ser más prolongada; mientras que quienes ingresan por intercambio estudian de un semestre hasta un año y más bien buscan tener nuevas experiencias académicas, sociales y culturales. En concordancia con este dato, el estudio halló que la mayor parte de estudiantes internacionales se encontraban realizando estudios graduados, mientras que la mayoría de intercambio se encontraban a nivel subgraduado. La edad de la mayoría fue representativa de las edades en las cuales la mayor parte de las personas realizan sus estudios universitarios para ingresar a la fuerza laboral. 
Para estudiantes estadounidenses puede resultar atractivo estudiar en Puerto Rico ya que tienen una experiencia cultural nueva, mientras permanecen en territorio estadounidense. Por su parte, los hispanos pueden estudiar en un lugar donde se habla su idioma, evitando así una de las principales dificultades que confrontan quienes tienen que aprender otro idioma por el país al que van a estudiar. Probablemente por esta razón, observamos que la mayoría de estudiantes del Programa Internacional que respondieron provienen de países hispanos, dato que contrasta con la población de estudiantes internacionales en los Estados Unidos, donde para el año académico 20092010, la mayoría era de países asiáticos (Institute of International Education, 2010).

El predominio del género femenino podría guardar relación con el hecho de que en los países de los cuales procede la mayoría (Colombia y Estados Unidos), la proporción de féminas realizando estudios universitarios es mayor que la de los hombres (Bonilla, López, Cintrón, Ramírez y Román, 2005; Mather y Adams, 2007). Por otro lado, la gran mayoría de estudiantes solteras y solteros puede coincidir con el hecho de que gran parte son personas jóvenes -entre 20 a 25 años de edad-, y se ha observado que muchos posponen sus planes de matrimonio, dando prioridad al desarrollo de una carrera, tendencia que documentaron estudios en los 90 y que en la actualidad se sostiene en otros contextos culturales (L. Novack y D. Novack, 1996; Romo, 2008; Spade y Reese, 1991); ello puede estar presentándose en la muestra, independientemente de las diferencias culturales que pueda haber entre los estudiantes internacionales y de intercambio. Por otro lado, debemos observar que es menos probable que estudiantes casadas o casados se hospeden en una residencia de la universidad, ya que en algunos casos optan por trasladar sus familias a Puerto Rico y estas no pueden vivir en las residencias de la Universidad de Puerto Rico.

Usualmente, los grupos de estudiantes que llevan menos tiempo son los que más necesidades presentan debido a su proceso de ajuste, siendo el periodo inicial de transición el más difícil (Poyrazli y Grahame, 2007). No obstante, observamos que los estudiantes con más tiempo mostraron necesidades que han permanecido latentes, ya que la mayoría de estos indicó tener las necesidades de conocer sobre los servicios estudiantiles, obtener orientación académica y desarrollar destrezas en el idioma inglés, entre otras, lo cual podría indicar que no han buscado estrategias de ayuda para atender estas necesidades. Relacionado con esto, una gran mayoría -incluyendo los estudiantes con estadía más prolongada- mostró interés en participar en una organización estudiantil. Una organización, además de trabajar por el bienestar de estos, tendría el aliciente de servir como red de apoyo social ante las dificultades que pueden confrontar.

\section{Necesidades académicas}

Una de las áreas donde más necesidades se identificaron fue la académica. Estas están vinculadas directamente al desempeño de su proceso de enseñanza-aprendizaje y pueden variar de acuerdo con la cultura, debido a los diferentes estilos y énfasis en la enseñanza y la evaluación. Un alto porcentaje señaló como muy importantes varias necesidades vinculadas al idioma. Esto responde al hecho de que muchos y muchas provienen de Estados Unidos y no dominan el idioma español, o provienen de países hispanoparlantes donde no se enfatiza la enseñanza del idioma inglés. Investigaciones anteriores han encontrado que el no dominar el idioma del país al que se va a cursar estudios es uno de los principales problemas de esta 
población estudiantil (Dorough, 2006; Gerbault, 2006; Sherry, Thomas y Chui, 2010; Wilton y Constantine, 2003; Yin, Huang y Hare, 2010).

Esta situación presenta un problema para quienes son estadounidenses, ya que el español es el idioma predominante en la enseñanza dentro de la Universidad de Puerto Rico; también resulta problemático para quienes no dominan el inglés, puesto que muchos libros de texto -particularmente en la Facultad de Ciencias Naturales y estudios graduados- son en este idioma. En ocasiones, los problemas relacionados con esto se agravan, ya que muchas veces no buscan ayuda debido a las diferencias por el lenguaje y la cultura, aun cuando se puede ver afectado su bienestar emocional (Bradley, 2000; Masuda et al., 2009; Mori, 2000; Zhang y Dixon, 2003). Esto provoca un impacto directo al área académica y afecta grandemente sus metas vocacionales y personales. En consecuencia, se afectan las posibilidades de éxito en un país extranjero donde las aulas universitarias tienen unas expectativas de graduar a estudiantes del extranjero como parte del mercadeo de esas instituciones y del mismo sistema globalizado.

Los servicios estudiantiles representan uno de los componentes más importantes durante el proceso académico. Relacionado con estos, la mayoría indicó tener necesidades de conocer los servicios estudiantiles, obtener orientación académica e información sobre los eventos académicos, servicios y facilidades universitarias, que podrían sugerir que no recibieron suficiente información o no asimilaron o entendieron la misma durante las orientaciones de nuevo ingreso dirigidas a esta población, así como dificultades en la comunicación y falta de conocimiento sobre cómo acceder a información de la universidad a través de los medios electrónicos, por lo que es importante que los estudiantes conozcan la página cibernética de la universidad y aprendan a utilizarla. Vinculado a esto, observamos que una gran cantidad indicó necesidades relacionadas al aprender a usar las bibliotecas, incluyendo el Internet y las redes de comunicación. Esta área es también de gran pertinencia ya que los trabajos bien fundamentados requieren destrezas de búsqueda así como conocimiento y acceso a las redes de revistas científicas y académicas, hallazgo que coincide con el estudio de Yi (2007).

También señalaron necesidades tales como aprender a preparar informes escritos y orales. Estas destrezas son sumamente importantes para el éxito académico, ya que estos trabajos forman parte de los medios de evaluación utilizados en muchos cursos. Estas necesidades no están limitadas a los estudiantes internacionales y de intercambio; Yoon y Portman (2004) han observado que muchos y muchas también presentan necesidades y problemas que son similares a los de estudiantes locales, por lo que ambos grupos pueden tener experiencias en común. No obstante, según hemos discutido, la mayoría de estudiantes internacionales y de intercambio no solicitan ayuda para sus necesidades y problemas (Bradley, 2000; Masuda et al., 2009; Mori, 2000; Zhang y Dixon, 2003), lo cual requiere una acción más proactiva por parte del personal universitario para trabajar estas y otras áreas de necesidad.

\section{Necesidades vocacionales}

Otra área donde los estudiantes y las estudiantes locales, internacionales y de intercambio tienen necesidades similares, es en el componente vocacional. Este es uno de suma importancia en el proceso de selección de carreras a nivel universitario, debido al impacto que tiene en el desarrollo 
académico y personal. Según Parsons (1909), en una sabia selección vocacional permean tres factores importantes: un claro entendimiento por parte de la persona de sí misma, aptitudes, habilidades, intereses, ambiciones, recursos, limitaciones, y sus causas; en segundo lugar, un conocimiento de los requisitos y condiciones de éxito, ventajas y desventajas, compensación, oportunidades, y perspectivas en diferentes líneas de trabajo; y en tercer lugar, un razonamiento certero de la relación entre los dos factores anteriores.

Muchos y muchas estudiantes señalaron como muy importantes varias necesidades vinculadas a esta área, lo cual plantea un desconocimiento sobre la carrera que se ha escogido y una gran necesidad de orientación al respecto. Esto concuerda con el marco teórico de Parsons y plantea la realidad vista en la experiencia de consejería de que muchos y muchas jóvenes inician estudios sin saber con certeza qué desean hacer en su vida profesional. También podría reflejar un desconocimiento del mundo globalizado desde el punto de vista económico y cómo esto ha impactado los escenarios de trabajos y las nuevas competencias cimentadas en la diversidad educativa que está exigiendo el mercado laboral.

Por otro lado, un alto porcentaje indicó tener la necesidad de conocer sobre internados, lo cual pudiera estar relacionado al hecho de que la mayoría se encontraba cursando estudios graduados, muchos de los cuales tienen este componente. Además, deseaban información sobre los ofrecimientos académicos en el recinto. Ello pudiera ser indicativo de dificultades en la comunicación y de un desconocimiento sobre la forma de acceder a esta información a través de los medios electrónicos en los que está disponible.

Relacionado con las ayudas económicas, observamos que cerca de una quinta parte de los estudiantes no recibe ayuda económica alguna, lo que puede dificultar el que alcance sus metas académicas. El gobierno de los Estados Unidos exige a aquellos y aquellas estudiantes con interés en realizar estudios en territorio estadounidense, el contar con $\$ 14,818$ para poder sufragar los gastos durante su estadía. Sin embargo, la mayoría indicó tener la necesidad de adquirir conocimientos sobre ayudas económicas disponibles en la universidad y conseguir un empleo a tiempo parcial dentro de la universidad, y además considera muy importante el que se trabaje con ambas necesidades. Esto concuerda con los hallazgos de Díaz (1992), cuya investigación es una de las pocas que ha tratado el tema de las necesidades y problemas de estudiantes internacionales en Puerto Rico y señala que esta área ha sido -y sigue siendo- una de sus principales preocupaciones. Los hallazgos de esta investigación puntualizan la necesidad respecto al conocer sobre ayudas económicas y generar ingresos con un empleo a tiempo parcial.

El recibir algún tipo de ayuda económica es particularmente importante para estudiantes internacionales ya que muchos y muchas, a diferencia de quienes provienen de Estados Unidos, no reciben ayudas económicas de sus gobiernos y reportan problemas financieros que pueden ser mayores que los de estudiantes locales (Hyun et al., 2007; Sherry et al., 2010). Además, debido al tipo de visa expedida, no pueden trabajar fuera de la universidad. Estudios han encontrado que aquellos y aquellas estudiantes que reciben algún tipo de ayuda, presentan menos problemas económicos (Sherry et al., 2010) y menos problemas de ajuste que quienes tienen que sufragar todos sus gastos (Lee, 2000); esto puntualiza la importancia de prestar atención a este asunto que resulta vital para el proceso académico y el bienestar general. 


\section{Necesidades personales /sociales}

Los estudiantes y las estudiantes internacionales y de intercambio pueden presentar necesidades personales y sociales particulares ya que, al estar insertados en un contexto físico y cultural ajeno al propio, no solo el entorno es diferente, sino también los vínculos sociales. En relación a estas, es necesario observar que, a pesar de que menos de la mitad indicó tener necesidades en esta área, un $50 \%$ o más marcó como muy importante el trabajar con dos necesidades: el manejo del tiempo y el ajuste social.

La necesidad de manejar el tiempo adecuadamente puede ser considerada como una compartida con muchos y muchas estudiantes del recinto. No obstante, las razones por las cuales los estudiantes y las estudiantes locales presentan esta necesidad pueden no ser las mismas que las de los grupos internacionales y de intercambio. En este caso, la razón puede estar más vinculada a las diferencias entre el país de procedencia de cada estudiante y Puerto Rico, las cuales pueden obligar a distribuir el tiempo de manera diferente para poder cumplir con las tareas. Un posible ejemplo de ello es la eficiencia en el sistema de transportación pública. Estudios han encontrado que cuando un o una estudiante procedente de un lugar que se caracteriza por contar con diversos y eficientes medios de transportación pública se va a estudiar a un lugar con un sistema menos eficiente o que simplemente carece de ello, confronta mayores dificultades en su proceso de ajuste (Poyrazli y Grahame, 2007; Yin et al., 2010). Ello implica una mayor inversión de tiempo y esfuerzo en lidiar con una situación que no confrontaba en su país.

El ajuste es una de las necesidades mayormente señaladas en estudios anteriores dadas las diferencias culturales (Gerbault, 2006; Mori, 2000; Sümer et al., 2008; Wilton y Constantine, 2003). Cuando hablamos de ajuste social, nos referimos a los cambios que se tienen que realizar para poder interactuar de forma adecuada con las personas del país recipiente. Un ejemplo de ello lo encontramos en la investigación de Laboy (2006), en la cual un estudiante internacional señaló que hay que desarrollar paciencia ante la burocracia y la lentitud, ya que en Puerto Rico está mal visto quejarse.

Aunque la mayoría de estudiantes internacionales y de intercambio no indicaron tener necesidades personales/sociales, sí consideraron importante trabajar con esta área. Respecto a esto, se podría interpretar que no presentan esta necesidad porque les ha resultado fácil el proceso de ajuste social en Puerto Rico; este hallazgo es comprensible al observar que la mayoría de los estudiantes del Programa Internacional provienen de países hispanos con los cuales tenemos elementos sociales y culturales en común, por lo que es posible que tengan satisfechas estas necesidades o que no las ubiquen como prioridad en su vida universitaria.

\section{Conclusiones y recomendaciones}

Cuando un o una estudiante se traslada de un país a otro, surgen necesidades que están relacionadas con el hecho de estar en un entorno físico y cultural diferente. Esto impacta de tal manera que se altera su proceso de vida a nivel académico, social, cultural e incluso emocional, y afecta áreas relacionadas con sus necesidades básicas. Este estudio identificó necesidades mayormente en las áreas académicas y vocacionales. Entre estas, sobresalen el 
aprender inglés o español, conocer los servicios estudiantiles y ofrecimientos académicos en Puerto Rico y Estados Unidos, explorar internados local e internacionalmente, y conocer sobre sueldos, tareas, escenarios y oportunidades relacionadas al empleo, así como ayudas económicas, por lo que se encontraron vigentes algunas necesidades señaladas por estudios anteriores (Díaz, 1992; Laboy, 2006). El que aún permeen necesidades que han sido identificadas anteriormente, puntualiza la necesidad de mayores esfuerzos por parte de la universidad para lograr atenderlas de manera más eficiente.

El idioma tuvo un papel de mucha relevancia en este estudio. En la Universidad de Puerto Rico, las clases por lo general son en español y muchos de sus libros de texto son en inglés, lo que representa una contradicción que podría dificultar la culminación de las metas académicas, situación más difícil aún para aquellos y aquellas cuyo primer idioma no es el inglés ni el español. Se añade a esto la existencia de innumerables palabras cuyos significados son distintos a otros países; la inserción de algunas palabras o frases del idioma inglés en el castellano por algunos y algunas estudiantes también puede afectar el mensaje que se está tratando de trasmitir, por lo que este interés en aprender el idioma español o inglés toma relevancia.

El estudio reflejó, además, el interés estudiantil por participar en una organización estudiantil que trabaje por su bienestar, oriente y sirva de apoyo en el proceso de ajuste al nuevo ambiente académico y social. Este ajuste podría estar afectado, pero sin limitarse a ello, por el hecho de que en esta residencia estudiantil se ubican, en su mayoría, estudiantes de post grado o que están culminando su bachillerato para insertarse en el mundo laboral. Por tal razón, están enfocados y enfocadas en la obtención de sus grados académicos en vez de tener un nivel de socialización más alto con estudiantes de otros países. Otro elemento que hay que añadir es que al DCODE asisten estudiantes internacionales y de intercambio afectados y afectadas emocionalmente, debido a que las metodologías utilizadas por los profesores y profesoras muchas veces no se ajustan al proceso de enseñanza-aprendizaje que han tenido en su país de origen.

El proceso académico debe estar unido a la prestación de servicios al estudiante como complemento de su desarrollo académico y vocacional. Sin embargo, aunque la universidad le ofrece una orientación de los mismos al llegar al país, tal parece que debe haber un seguimiento más directo y continuo debido a la importancia que los estudiantes y las estudiantes internacionales le asignan a este aspecto en esta investigación. La orientación de todos los servicios en la universidad, generalmente se realiza en la primera semana de estadía en el país, por lo que es posible suponer que están explorando su entorno y tratando de conocer personas y lugares, y queda en un segundo nivel la importancia de los mismos para el éxito de sus metas trazadas.

Es importante señalar que parte del esfuerzo de estos grupos de estudiantes radica en lograr el ajuste al nuevo país, por lo que no es hasta que comienzan las clases que sienten el choque a nivel cultural, de metodologías de la enseñanza y las exigencias académicas. Esto afecta el que puedan aprender la forma y manera en que se espera que el estudiante en formación presente los informes escritos y orales. Estas destrezas son de mucha importancia para el manejo emocional de sentimientos de logros.

Al área académica se sumó la vocacional como una de importancia. Cuando ofrecemos el proceso de consejería de carreras nos percatamos que no están claros y claras en relación con los factores a considerar. Entre ellos se destacan: el nivel de vida que en un futuro quieren tener; si tendrán las capacidades de desarrollar las destrezas, habilidades y aptitudes que exige la ocupación; 
el hecho de que puedan ejercer la misma en diferentes partes del mundo; la disponibilidad de oferta y demanda; y las exigencias de un mundo globalizado donde las personas profesionales que tengan mayor diversidad educativa y laboral, serán posiblemente a quienes recluten.

Al realizar el análisis en cuanto al área académica y vocacional, el factor económico se hace importante e imprescindible. Por los hallazgos de este estudio, tal parece que desconocen el impacto económico que surge al estar en un país diferente al suyo y el efecto de no poder trabajar por el tipo de visado que tienen. Esto les ha creado un sinnúmero de problemas, ya que en ocasiones desean quedarse para realizar otros grados o especialidades y económicamente les resulta difícil. Los hallazgos de esta investigación sugieren la necesidad de mayor orientación en relación con ayudas económicas y ayudantías de cátedra dentro de la misma universidad.

Por otro lado, el hecho de que la mayoría de estudiantes no indicaran tener necesidades de índole personal/social podría sugerir mayores semejanzas que diferencias en términos culturales y sociales, sobre todo al pensar en la gran cantidad de estudiantes de habla hispana en el recinto, así como un entorno manejable por el uso de libros en inglés y la familiaridad de usar una misma moneda al pensar en los grupos de estadounidenses.

El proceso de identificar necesidades no está completo si no se definen las estrategias y recursos que se utilizarán para trabajar con las mismas al entender, al igual que otras y otros que han abordado el tema, que la población estudiantil internacional y de intercambio se podrá desarrollar de manera exitosa en la medida en que tenga un sistema de apoyo en su entorno académico (Gu et al., 2010). Por tal razón, presentamos recomendaciones para atender las necesidades que los estudiantes y las estudiantes indicaron tener y que señalaron como muy importante el que se trabaje con las mismas.

En relación con las necesidades académicas, recomendamos el desarrollo de un Programa de Tutorías de Pares para que aprendan inglés o español. Las tutorías con pares representan una forma alternativa de enseñanza y han evidenciado ser efectivas en la transmisión de conocimiento; además, estudios han encontrado que la mayoría de estudiantes disfrutan aprender de sus pares (Keller, 2009; Tessier, 2007). Además, podría servirles como un recurso de apoyo en el proceso de ajuste, ya que muchas veces prefieren hablar sobre sus problemas con pares antes de buscar ayuda profesional o hablar con un supervisor. También se les puede ayudar en la coordinación con lugares donde puedan aprender idiomas, de acuerdo con la necesidad de cada uno. El dominio del idioma es sumamente importante, ya que los estudios resaltan que estudiantes internacionales exitosos típicamente aprenden el idioma local e incorporan mayores estrategias de aprendizaje como parte de su aculturación académica (Cheng y Fox, 2008).

Es sumamente importante que las instituciones educativas provean orientación. Para ello, se debe contar con profesionales bilingües de ayuda, de manera que se pueda orientar al estudiante en el idioma que mejor entienda y se pueda atender las necesidades de forma adecuada. Asimismo, el personal de ayuda de diversas ramas - psicología, trabajo social, consejería, consejería en rehabilitación, etc.- debe informar a los estudiantes sobre las oficinas y teléfonos a través de los cuales se les puede contactar y especificar los servicios de ayuda que pueden recibir a través de ellas y ellos.

Para atender la necesidad encontrada sobre orientación de servicios estudiantiles, se debe coordinar con el Decanato de Asuntos Estudiantiles de la Universidad de Puerto Rico para que se les ofrezca la misma. Según discutimos, es fundamental que los estudiantes y las estudiantes sepan hacer uso de los sistemas de bibliotecas, incluyendo los recursos electrónicos disponibles que les 
sirven de apoyo para realizar sus trabajos. Por ello, las instituciones deben asegurarse de ofrecer talleres sobre el uso de los sistemas bibliotecarios. En el caso de la Universidad de Puerto Rico, se debe orientar también sobre el uso de la página electrónica de la universidad a través de la cual se puede acceder a una gran cantidad de información de pertinencia disponible en distintos idiomas. Por otro lado, se pueden ofrecer talleres sobre la preparación de informes orales y escritos a través del Centro de Excelencia Académica.

Respecto a las necesidades vocacionales, las instituciones deben ayudar a los estudiantes y las estudiantes a definir qué desean en términos de su vida profesional, de manera que no pierdan tiempo estudiando en un campo que realmente no les interesa o satisface como esperaban. Para esto, el DCODE puede ayudar a explorar intereses vocacionales y orientar sobre los ofrecimientos académicos en Puerto Rico y Estados Unidos. Respecto al conocer sobre sueldo, escenarios y oportunidades de empleo a nivel internacional, este departamento debe ofrecerles orientaciones tomando como referencia el $\mathrm{O}$, net, el cual es un diccionario de títulos ocupacionales que contiene esta información. Por otra parte, las instituciones pueden coordinar con la organización estudiantil "AIESEC" (Association Internationale des Etudiants en Sciences Economiques et Commerciales) para que los estudiantes y las estudiantes internacionales reciban orientación relacionada con oportunidades de internado; en la Universidad de Puerto Rico, el Decanato de Estudios Graduados e Investigación (DEGI) puede ofrecer esta información en coordinación con el DCODE.

Como hemos puntualizado, es muy importante que quienes no reciben ayuda económica de sus países puedan recibir alguna ayuda, ya sea a través de becas o de ayudantías como asistencia de cátedra o de investigación. Para ello, el DCODE debe coordinar una orientación con la Oficina de Asistencia Económica y el DEGI de manera que los estudiantes y las estudiantes conozcan sobre ayudas económicas disponibles, oportunidades de ingresos dentro de la universidad como estudiante internacional y sus requisitos. Además, es importante que reciban orientación sobre las leyes que les aplican y las visas que deben solicitar para poder trabajar o recibir ayudas económicas, entre otros aspectos.

Finalmente, para ayudar a manejar el tiempo adecuadamente, el DCODE debe organizar talleres en los que puedan conocer las herramientas que les permitirán desarrollar esta destreza considerando el contexto puertorriqueño. Asimismo, debe coordinar un taller en el que participen estudiantes locales de forma voluntaria para ayudar a estudiantes internacionales y de intercambio con los ajustes sociales. Resulta necesario que todos los talleres sean anunciados a través de diversos medios como hojas sueltas y la página electrónica de la universidad. Además, se les puede enviar mensajes a sus correos electrónicos para que se les notifique sobre los mismos.

El aumento de estudiantes internacionales y de intercambio junto con las políticas de internacionalización, tanto en Puerto Rico como en otros países, exige que se conozcan y se atiendan las necesidades de estos grupos estudiantiles. Esta investigación representó un esfuerzo hacia ese camino. El hecho de que estudios anteriores en el Recinto de Río Piedras hayan señalado necesidades y problemas que continúan vigentes (Díaz, 1992; Laboy, 2006) recalca la urgencia de que se gesten acciones y colaboraciones, dentro de la universidad, que trabajen para atender sus necesidades, de manera que sea más viable el alcanzar las metas académicas y aspirar a la autorrealización. 


\section{Referencias bibliográficas}

Afflick, B. (2009). International Students' Perception of their Undergraduate Experience [Percepción de los estudiantes internacionales de su experiencia subgraduada] (Disertación doctoral). Recuperada de la base de datos ProQuest Dissertations and Theses. (UMI No. 3360208)

Bonilla, V., López, A., Cintrón, M., Ramírez, S. y Román, R. (2005, diciembre). Feminización de la matrícula de educación superior en Puerto Rico. Cuaderno de Investigación en la Educación, 20. Recuperado de http://cie.uprrp.edu/cuaderno/ediciones/20/pdf/c20art7.pdf

Bradley, G. (2000). Responding Effectively to the Mental Health Needs of International Students [Respondiendo efectivamente a las necesidades de salud mental de los estudiantes internacionales]. Higher Education, 39(4), 417-433.

Cemalcilar, Z. y Falbo, T. (2008, noviembre). A Longitudinal Study of the Adaptation of International Students in the United States [Un estudio longitudinal sobre la adaptación de los estudiantes internacionales en los Estados Unidos]. Journal of Cross-Cultural Psychology, 39(6), 799-804. doi: 10.1177/0022022108323787

Charles-Toussaint, G. C. y Crowson, H. M. (2010, octubre). Prejudice Against International Students: The Role of Threat Perceptions and Authoritarian Dispositions in U.S. Students [Prejuicio contra estudiantes internacionales: El rol de las percepciones de amenazas y disposiciones autoritarias en estudiantes de Estados Unidos]. The Journal of Psychology, 144(5), 413-428.

Cheng, L. y Fox, J. (2008, diciembre). Towards a Better Understanding of Academic Acculturation: Second Language Students in Canadian Universities [Hacia un mejor entendimiento de la aculturación académica: Estudiantes con segundo idioma en universidades canadienses]. The Canadian Modern Language Review, 65(2), 307-333. doi: 10.3138/cmlr.65.2.307

Díaz, L. M. (1992). Necesidades y problemas de los estudiantes internacionales matriculados en el Recinto de Río Piedras de la Universidad de Puerto Rico (Tesis de maestría no publicada). Universidad de Puerto Rico, Río Piedras.

Dorough, C. (2006). Evaluation of Academic and Social Needs of Foreign-Born Students at a University and School of Nursing [Evaluación de las necesidades académicas y sociales de estudiantes nacidos en el extranjero en una universidad y escuela de enfermería]. (Disertación doctoral). Recuperada de la base de datos ProQuest Dissertations and Theses. (UMI No. 3220870)

Erichsen, E. A. y Bollinger, D. U. (2011). Towards Understanding International Graduate Student Isolation in Traditional and Online Environments [Hacia el entendimiento del aislamiento de 
los estudiantes internacionales graduados en ambientes tradicionales y en línea]. Education Technology Research Development, 59(3), 309-326. doi: 10.1007/s11423-010-9161-6

Gerbault, J. (2006). Needs Analysis as a Tool for Supporting Student Mobility [Análisis de necesidades como una herramienta para apoyar la movilidad estudiantil]. Trabajo presentado en la International Bilingual Conference on Academic Mobility: Crossed Perspectives, Finland. Recuperado de http://www.euro-mobil.org/publications/publ NeedsAnalysisFR.pdf

Gu, Q., Schweisfurth, M., y Day, C. (2010, enero). Learning and Growing in a 'Foreign' Context: Intercultural Experiences of International Students [Aprender y crecer en un contexto 'extranjero': Experiencias interculturales de estudiantes internacionales]. Compare, 40(1), 7-23. doi: 10.1080/03057920903115983

Hernández, R., Fernández, C., y Baptist, P. (2003). Metodología de la investigación (2ª ed.). México: McGraw-Hill.

Hwang, K. P., Wang, M.-K. y Sodanine, S. (2011). The Effects of Stressors, Living Support, and Adjustment on Learning Performance of International Students in Taiwan [Los efectos de los estresores, apoyo al diario vivir y ajuste sobre el desempeño del aprendizaje de los estudiantes internacionales en Taiwán]. Social Behavior and Personality, 39(3), 333-344. doi: 10.2224/ sbp.2011.39.3.333

Hyun, J., Quinn, B., Madon, T. y Lustig, S. (2007). Mental Health Need, Awareness, and Use of Counseling Services Among International Graduate Students [Necesidad de salud mental, conocimiento y uso de los servicios de consejería entre estudiantes internacionales graduados]. Journal of American College Health, 56(2), 109-118. doi: 10.3200/JACH.56.2.109-118

Institute of International Education. (2010). Open Doors 2010: International Students in the United States [Puertas abiertas 2010: Estudiantes internacionales en los Estados Unidos]. Recuperado de http://www.iie.org/Research-and-Publications/Open-Doors/ /media/Files/ Corporate/Open-Doors/Fast-Facts/Fast\%20Facts\%202010.ashx

Keller, S. D. (2009). Validation of Peer-Teaching-Peer Paraprofessional Model in Teen Parenting Nutrition Education [Validación del modelo para-profesional de pares enseñando a pares en la educación de nutrición para padres y madres adolescentes] (Tesis de maestría). Disponible en la base de datos ProQuest Dissertations and Theses. (UMI No. 1465567)

Laboy, B. (2006). Perceptions of university counseling services to foreign students [Percepción sobre los servicios de consejería al estudiante extranjero universitario] (Disertación doctoral). Disponible en la base de datos ProQuest Dissertations and Theses. (UMI No. 3220407)

Lee, K. W. (2000). A Comparative Analysis of Foreign Student Needs in Taiwan [Un análisis comparativo de las necesidades de estudiantes extranjeros en Taiwán]. (Tesis de maestría, 
Hsuan Chuang University, Taiwan). Recuperado de http://library.hcu.edu.tw/ezcatfiles/b048/ img/img/424/CAS0202.pdf

Maslow, A. H. (1967). Motivación y personalidad. España: Ediciones Sagitario.

Masuda, A., Hayes, S. C., Twohig, M. P., Lillis, J., Fletcher, L. B., \& Gloster, A. T. (2009). Comparing Japanese International College Students' and U.S. College Students' Mental-Health-Related Stigmatizing Attitudes [Comparando las actitudes estigmatizantes relacionadas a salud mental entre estudiantes universitarios japoneses y estudiantes universitarios estadounidenses]. Journal of Multicultural Counseling and Development, 37(3), 178-189.

Mather, M. y Adams, D. (2007). The Crossover in Female-Male College Enrollment Rates [El cruce en las tasas de matrícula universitaria entre féminas y varones]. Population Reference Bureau. Recuperado de http://www.prb.org/Articles/2007/ CrossoverinFemaleMaleCollegeEnrollmentRates.aspx

Mori, S. (2000, Spring). Addressing the Mental Health Concerns of International Students [Respondiendo a las preocupaciones de salud mental de estudiantes internacionales]. Journal of Counseling \& Development, 78(2), 137-144.

Nilsson, J. E., Butler, J., Shouse, S. y Joshi, C. (2008, Fall). The Relationships Among Perfectionism, Acculturation, and Stress in Asian International Students [Las relaciones entre perfeccionismo, aculturación y estrés en estudiantes internacionales asiáticos]. Journal of College Counseling, 11(2), 147-158.

Novack, L. L. y Novack, D. R. (1996, julio). Being Female in the Eighties and Nineties: Conflicts Between New Opportunities and Traditional Expectations Among White, Middle Class, Heterosexual College Women [Ser fémina en los ochentas y noventas: Conflictos entre nuevas oportunidades y expectativas tradicionales entre mujeres universitarias heterosexuales, blancas y de clase media]. Sex Roles, 35(1-2), 57-77.

Parsons, F. (1909). Choosing a Vocation [La elección de una vocación]. Boston: Houghton Mifflin. Recuperado de http://www.archive.org/stream/choosingavocati00parsgoog - page/n8/ mode/2up

Poyrazli, S. y Grahame, K. M. (2007). Barriers to Adjustment: Needs of International Students Within a Semi-Urban Campus Community [Barreras al ajuste: Necesidades de estudiantes internacionales dentro de una comunidad semi-urbana del campus]. Journal of Instructional Psychology, 34(1), 28-45.

Romo, J. M. (2008). Estudiantes universitarios y sus relaciones de pareja: De sus experiencias y proyectos de vida. Revista Mexicana de Investigación Educativa, 13(38), 801-823. 
Russell, J., Rosenthal, D. y Thomson, G. (2010). The International Student Experience: Three Styles of Adaptation [La experiencia del estudiante internacional: Tres estilos de adaptación]. Higher Education, 60(2), 235-249. doi: 10.1007/s10734-009-9297-7

Sherry, M., Thomas, P. \& Chui, W. H. (2010). International Students: A Vulnerable Student Population [Estudiantes internacionales: Una población estudiantil vulnerable]. Higher Education, 60(1), 33-46. doi: 10.1007/s10734-009-9284-Z

Spade, J. Z. y Reese, C. A. (1991). We've Come a Long Way, Maybe: College Students' Plans for Work and Family [Hemos recorrido un largo camino, tal vez: Planes de los estudiantes universitarios para el trabajo y la familia]. Sex Roles, 24(5-6), 309-321.

Suanet, I. y Van de Vijver, F. J. R. (2009, mayo-junio). Perceived Cultural Distance and Acculturation Among Exchange Students in Russia [Distancia cultural percibida y aculturación entre estudiantes de intercambio en Rusia]. Journal of Community \& Applied Social Psychology, 19(3), 182-197. doi: 10.1002/casp.989

Sümer, S., Poyrazli, S. y Grahame, K. (2008, Fall). Predictors of Depression and Anxiety Among International Students [Predictores de depresión y ansiedad entre estudiantes internacionales]. Journal of Counseling \& Development, 86(4), 429-437.

Szelényi, K. (2006, Fall). Students Without Borders? [¿Estudiantes sin fronteras?] Migratory Decision-Making Among International Graduate Students in the U.S. [Toma de decisiones relacionadas a migración entre estudiantes graduados internacionales en los Estados Unidos] Knowledge, Technology, \& Policy, 19(3), 64-86. doi: 10.1007/s12130-006-1030-6

Tessier, J. (2007, enero-febrero). Small-Group Peer Teaching in an Introductory Biology Classroom [La enseñanza en pequeños grupos de pares en un aula de introducción a la biología]. Journal of College Science Teaching, 36(4), 64-69.

Tomich, P. C., McWhirter, J. J. y Darcy, M. (2003). Personality and International Students' Adaptation Experience [Personalidad y experiencia de adaptación de estudiantes internacionales]. International Education, 33(1), 22-39.

Universidad de Puerto Rico, Oficina de Planificación Académica. (2009). Distribución de estudiantes extranjeros y no residentes por nivel de estudios y género (Reporte sin publicar). Universidad de Puerto Rico, Río Piedras.

Väfors, M., Chin, D. y DeMarinis, V. (2008). Stressors, Anxiety, Acculturation and Adjustment Among International and North American students [Estresores, ansiedad, aculturación y ajuste entre estudiantes internacionales y estudiantes norteamericanos]. International Journal of Intercultural Relations, 32(3), 244-259. doi: 10.1016/j.ijintrel.2008.01.001 
Vélez, M. (2006). Cuestionario de necesidades de orientación de los estudiantes de primer año. Departamento de Consejería para el Desarrollo Estudiantil (DCODE) de la Universidad de Puerto Rico. Instrumento sin publicar.

Wadsworth, B. C., Hecht, M. L. y Jung, E. (2008). The role of identity gaps, discrimination, and acculturation in international students' educational satisfaction in American classrooms [El rol de las lagunas en la identidad, discrimen y aculturación en la satisfacción de los estudiantes internacionales en relación a las aulas americanas]. Communication Education, 57(1), 64-87. doi: $10.1080 / 03634520701668407$

Wilton, L. y Constantine, M. G. (2003). Length of Residence, Cultural Adjustment Difficulties, and Psychological Distress Symptoms in Asian and Latin American International College Students [Tiempo de residencia, dificultades en la adaptación cultural y síntomas de angustia psicológica en estudiantes universitarios internacionales asiáticos y latinoamericanos]. Journal of College Counseling, 6(2), 177-186.

Yin, L., Huang, L.-C. y Hare, D. (2010). Perspectives of South Korean Undergraduate Exchange Students Attending a University in the Southern United States [Perspectivas de los estudiantes surcoreanos subgraduados de intercambio asistiendo a una universidad en el sur de los Estados Unidos]. Journal of The First-Year Experience \& Students in Transition, 22(2), 93-111.

Ying, Y.-W. y Han, M. (2006, septiembre). The Contribution of Personality, Acculturative Stressors, and Social Affiliation to Adjustment: A Longitudinal Study of Taiwanese Students in the United States [La contribución de la personalidad, los estresores de aculturación y la afiliación social al ajuste: Un estudio longitudinal de estudiantes taiwaneses en los Estados Unidos]. International Journal of Intercultural Relations, 30(5), 623-635. doi: 10.1016/j.ijintrel.2006.02.001

Yi, Z. (2007). International Student Perceptions of Information Needs and Use [Percepciones de los estudiantes internacionales de las necesidades y uso de información]. The Journal of Academic Librarianship, 33(6), 666-673. doi: 10.1016/j.acalib.2007.09.003

Yoon, E. y Portman, T. A. (2004, enero). Critical Issues of Literature on Counseling International Students [Los temas críticos de la literatura en la consejería a estudiantes internacionales]. Journal of Multicultural Counseling and Development, 32(1), 33-44.

Zhang, N. y Dixon, D. N. (2003). Acculturation and Attitudes of Asian International Students Toward Seeking Psychological Help [Aculturación y actitudes de los estudiantes internacionales asiáticos hacia la búsqueda de ayuda psicológica]. Journal of Multicultural Counseling and Development, 31(3), 205-222. 